\title{
Harmonic-Rejection Compact Bandpass Filter Using Defected Ground Structure for GPS Application
}

\author{
Haiwen Liu, ${ }^{1,2}$ Baoping Ren, ${ }^{2}$ Xiang Xiao, ${ }^{2}$ Zhichong Zhang, ${ }^{2}$ Shen $\mathrm{Li}^{2}{ }^{2}$ and Suping Peng ${ }^{1}$ \\ ${ }^{1}$ State Key Laboratory of Coal Resources and Safe Mining, China University of Mining \& Technology, Beijing 100083, China \\ ${ }^{2}$ College of Information Engineering, East China of Jiaotong University, Nanchang 330013, China \\ Correspondence should be addressed to Haiwen Liu; liuhaiwen@gmail.com
}

Received 31 December 2013; Accepted 14 March 2014; Published 3 April 2014

Academic Editor: Huikai Xie

Copyright (c) 2014 Haiwen Liu et al. This is an open access article distributed under the Creative Commons Attribution License, which permits unrestricted use, distribution, and reproduction in any medium, provided the original work is properly cited.

\begin{abstract}
A miniaturized bandpass filter (BPF) using defected ground structure (DGS) resonator with the characteristic of harmonic rejection is developed in this paper. The second and third harmonics of the proposed BPF are rejected by the characteristic of steppedimpedance DGS resonator. Moreover, open stubs are established so that two adjustable transmission zeros can independently be created to extend the stopband and improve the rejection level. Finally, a second-order BPF, centered at $1.62 \mathrm{GHz}$ with a stopband extended up to $5.6 \mathrm{GHz}$ and a rejection level better than $20 \mathrm{~dB}$, is designed and implemented for GPS application. A good agreement between simulation and measurement verifies the validity of this design methodology.
\end{abstract}

\section{Introduction}

Miniaturized high-performance narrow-band microwave bandpass filters (BPFs) are highly desirable for the next generation of satellite and mobile communications systems [1,2]. However, these microstrip BPFs with uniform-impedance resonators usually suffer from the spurious passbands at the multiple fundamental frequencies. Therefore, the spurious passbands will cause the upper stopband performance worse. To solve this problem, much effort has been made to suppress the spurious passbands [3-8]. In [3], the "wiggly-line" filters using a sinusoidally varying linewidth were proposed to give an effective suppression on the first spurious response around $2 f_{0}$. By etching split-ring resonators to achieve strong magnetic coupling, the first spurious passband may also be suppressed [4]. In recent years, stepped-impedance resonators (SIRs) are applied to shift the higher order mode, while some approaches also use the higher frequencies to suppress the harmonics $[5,6]$. By using suitable impedance and electrical length ratio, the higher order mode can be pushed to higher frequency [5]. An enhanced stepped-impedance combline $\mathrm{BPF}$ is with a tapped transformer coupling at input and output [6]. In addition, the wide-stopband BPF with quarterwavelength SIRs has been proposed [7]. To further improve the stopband performance, the resonators with embedded bandstop structures are used to suppress the lower spurious harmonics of SIRs [8]. Thus, harmonic suppression can be carried out by the ground-plane aperture [9].

In this paper, a novel design of harmonic rejection BPF with stepped-impedance defected ground structure (DGS) resonator is proposed. The stepped-impedance DGS structure can be realized to suppress the first and the second harmonics because two attenuation poles are introduced within the upper passband to extend the stopband of the proposed filter. Finally, a second-order BPF using $\lambda / 2$ stepped-impedance DGS resonator is designed, analyzed, and fabricated. Simulations and measurements validate the proposed design principle.

\section{Materials and Methods}

As illustrated in Figure 1, the proposed BPF filter consists of top layer and bottom layer. A couple of meandering microstrip feed lines are located on the top one. On the bottom layer, a pair of stepped-impedance hairpin DGS resonators is etched on the backside metallic ground plane. Figure 2 shows the top view of the proposed filter, where 

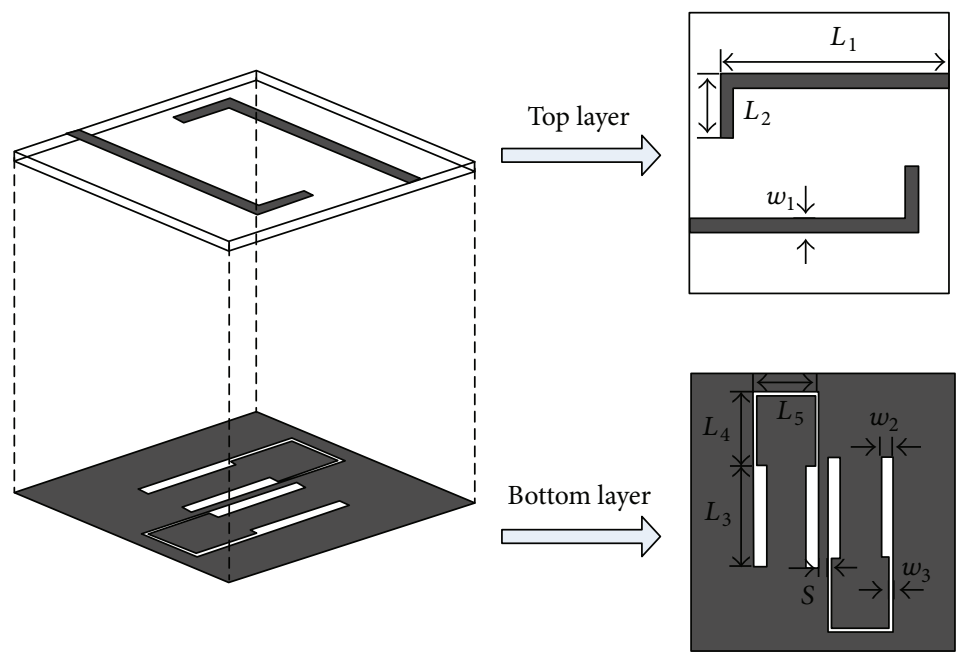

FIGURE 1: Schematic views of the proposed filter.

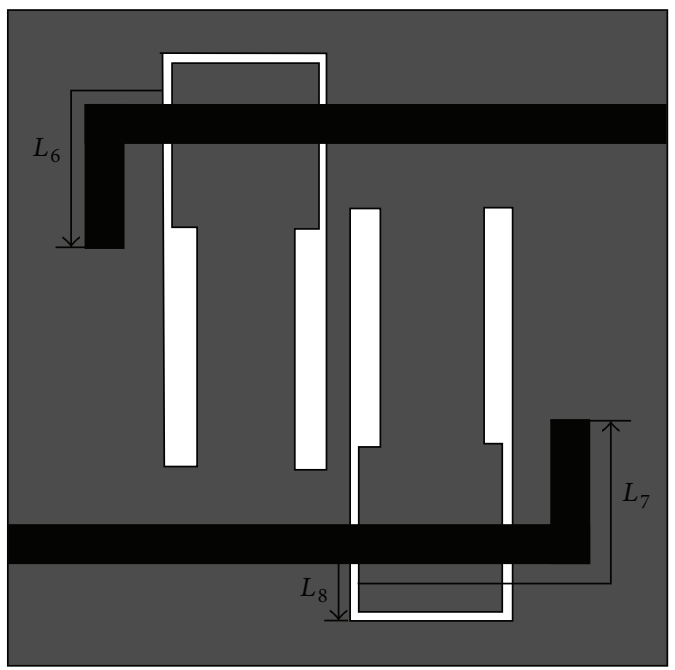

FIGURE 2: Top view of the proposed DGS filter.

the DGS resonators are excited by the meandering microstrip feed lines. The coupling between microstrip feed lines and DGS resonator is realized by the microstrip-to-slotline transition structure, which also provides two open stubs $\left(L_{6}\right.$, $L_{7}$ ) for producing two transmission zeros. In addition, the length of each stub is properly being designed to $\lambda / 4$, which is operated at the unwanted frequency $f_{t}$. Therefore, a transmission zero would be created to suppress the unwanted harmonics at $f_{t}$. For the purpose of impedance matching, the impedance of the open stub is designed at $50 \Omega$.

A layout of the proposed stepped-impedance DGS resonator is attached in Figure 3, where the resonator has different characteristic impedances that are indicated with $Z_{1}$ and $Z_{2}$ and their corresponding electrical lengths are $\theta_{1}$ and $\theta_{2}$, respectively. Its input admittance viewed from the left port

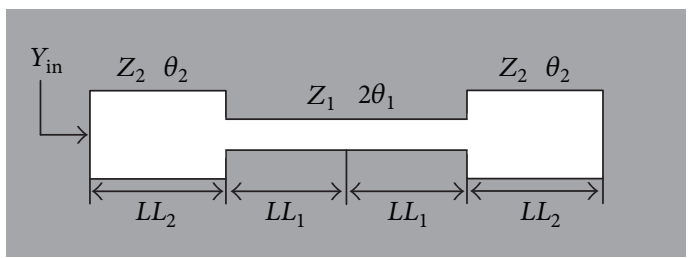

FIGURE 3: Layout of the proposed stepped-impedance DGS resonator.

can be derived by means of the transmission line theory as follows:

$$
\begin{aligned}
Y_{\text {in }}=j Y_{2}( & \left(2\left(K \tan \theta_{1}+\tan \theta_{2}\right)\right. \\
& \left.\cdot\left(K-\tan \theta_{1} \tan \theta_{2}\right)\right) \\
\times & \left(K\left(1-\tan ^{2} \theta_{1}\right)\left(1-\tan ^{2} \theta_{2}\right)\right. \\
& \left.\left.-2\left(1+K^{2}\right) \tan \theta_{1} \tan \theta_{2}\right)\right),
\end{aligned}
$$

where impedance ratio $K=Z_{2} / Z_{1}$.

Let $Y_{\text {in }}=0$; the resonant conditions can be obtained as follows:

$$
\begin{gathered}
\left(K \tan \theta_{1}+\tan \theta_{2}\right) \cdot\left(K-\tan \theta_{1} \tan \theta_{2}\right)=0, \\
K=\tan \theta_{1} \tan \theta_{2},
\end{gathered}
$$

where $\theta_{1}=\beta L L_{1}, \theta_{2}=\beta L L_{2}$, and $L L_{1}$ and $L L_{2}$ are the physical dimension of the stepped-impedance DGS resonator.

To explain the unique harmonic suppression characteristic of the stepped-impedance DGS resonator, the corresponding simulation under the condition of weak coupling has been demonstrated. The responses of a stepped-impedance DGS resonator versus the different impedance ratio, $K$, under the fixed condition of $\theta_{1}=\theta_{2}$ are depicted in Figure 4. It 


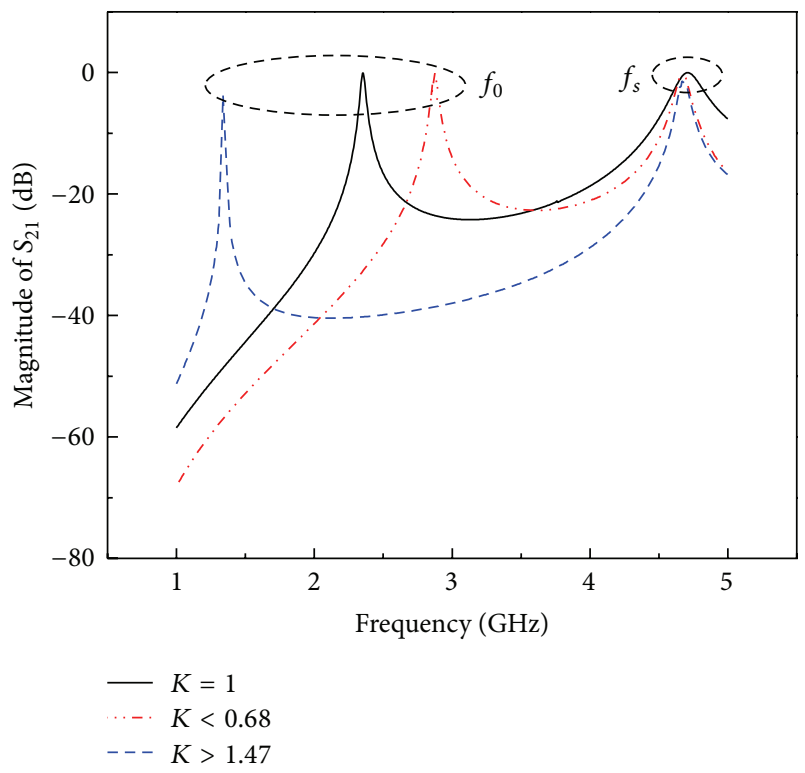

FIGURE 4: The fundamental frequency varied with the impedance ratio, $K$. $\left(\theta_{1}=\theta_{2}\right)$.

can be observed that when the second harmonic frequency, $f_{s}$, is set at $4.8 \mathrm{GHz}$, the fundamental frequency, $f_{0}$, will be shifted from 2.4 to $2.88 \mathrm{GHz}$ when $K=0.68(<1)$, and $f_{0}$ will be decreased from 2.4 to $1.34 \mathrm{GHz}$ when $K=1.47(>1)$, resulting in the frequency radio $f_{s} / f_{0}$ larger than 3 , such that the second and third harmonics will be rejected. Therefore, when impedance radio of the stepped-impedance DGS resonator is chosen greater than 1 , a wide range stopband can be obtained easily.

\section{Results and Discussion}

Based on the above analysis, a second-order harmonicrejected BPF with the proposed stepped-impedance DGS resonator was designed and fabricated for GPS application. Taconic-RF-35A2 substrate with a relative dielectric constant of 3.5 and a thickness of $0.76 \mathrm{~mm}$ was used. Also both sides of the substrate are coated with copper. The feed lines are connected to $50 \Omega$ microstrip lines with a width of $1.7 \mathrm{~mm}$. The other physical parameters are calculated and optimized as follows: $L_{1}=28.5 \mathrm{~mm}, L_{2}=7 \mathrm{~mm}, L_{3}=12 \mathrm{~mm}$, $L_{4}=8 \mathrm{~mm}, L_{5}=6.7 \mathrm{~mm}, L_{6}=8.8 \mathrm{~mm}, L_{7}=15.5 \mathrm{~mm}$, $L_{8}=4.6 \mathrm{~mm}, w_{1}=1.7 \mathrm{~mm}, w_{2}=1.8 \mathrm{~mm}, w_{3}=0.4 \mathrm{~mm}$, and $s=0.5 \mathrm{~mm}$. The photographs of the fabricated filter are shown in Figure 5.

The designed circuit was simulated by the commercial simulator, Ansoft HFSS 10, and the fabricated one is measured by network analyzer of CETC AV3629. Its simulated and measured frequency responses are compared in Figure 6. It is observed that two poles are located at 1.56 and $1.69 \mathrm{GHz}$, respectively. The $3 \mathrm{~dB}$ fractional bandwidth, from 1.47 to $1.76 \mathrm{GHz}$, is $18 \%$ and its return loss in the passband is less than $-18 \mathrm{~dB}$, which can be applied to GPS $(1.575 \mathrm{GHz})$ properly. In addition, there are three transmission zeros, that is, $\mathrm{TZ}_{1}$, $\mathrm{TZ}_{2}$, and $\mathrm{TZ}_{3}$, on the upper stopband, which are $-30 \mathrm{~dB}$,

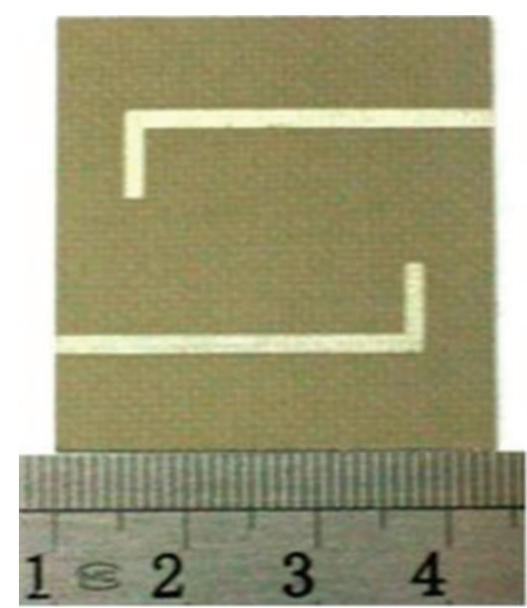

(a) Top view

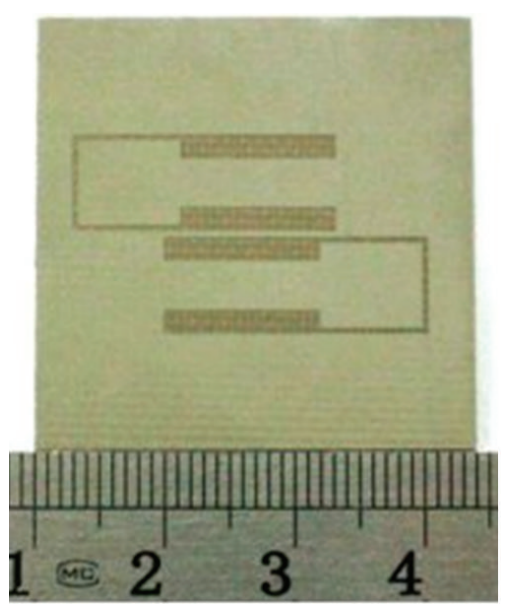

(b) Bottom view

FIGURE 5: Photograph of the proposed filter. (a) Top view and (b) bottom view.

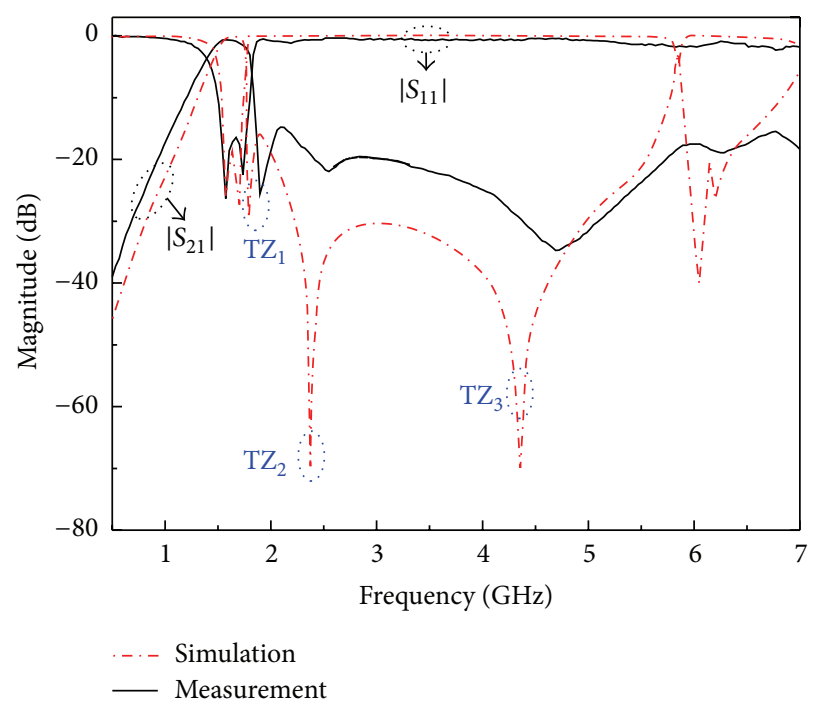

FIGURE 6: Measured and simulated response of the proposed filter. 
$-70 \mathrm{~dB}$, and $-69 \mathrm{~dB}$ at frequencies of $1.8 \mathrm{GHz}, 2.45 \mathrm{GHz}$, and $4.33 \mathrm{GHz}$, respectively, thus extended the stopband, and enhanced the rejection level. Especially, the transmission zero at $1.8 \mathrm{GHz}$ is close to the passband edges and can eliminate the impact of GSM systems, which greatly improve the selectivity of the filter. The measured minimum insertion loss achieves $0.73 \mathrm{~dB}$ in the passband. Measured results agree well with the simulated ones and prove the validity of the introduced design principles to produce the resonant characteristics and harmonic rejection. Some discrepancy between the measurement and simulation can be attributed to the inaccuracy in fabrication and implementation.

\section{Conclusions}

A novel design of harmonic-rejected BPF with steppedimpedance DGS resonator is proposed for GPS application. The pair of stepped-impedance DGS resonators are excited by a couple of folded microstrip feed lines. The proposed $\mathrm{BPF}$ has the advantage of wide-band harmonic rejection. Furthermore, open stubs are used to produce multiple adjustable transmission zeros which are applied to improve the rejection level. Finally, the proposed filter with slotline structure has improved power-carrying capacity for potential high-power application.

\section{Conflict of Interests}

The authors declare that there is no conflict of interests regarding the publication of this paper.

\section{Acknowledgments}

This work was supported by the National Science Foundation of China (nos. 61061001 and 61161005) and the International Cooperation Funds and Science and Technology Innovation Team, Jiangxi, China (nos. 20121BDH80015 and 20122BCB24025).

\section{References}

[1] J. S. Hong and M. J. Lancaster, "Microstrip bandpass filter using degenerate modes of a novel meander loop resonator," IEEE Microwave and Guided Wave Letters, vol. 5, no. 11, pp. 371-372, 1995.

[2] S.-Y. Lee and C. M. Tsai, "New cross-coupled filter design using improved hairpin resonators," IEEE Transactions on Microwave Theory and Techniques, vol. 48, no. 12, pp. 2482-2490, 2000.

[3] T. Lopetegi, M. A. G. Laso, J. Hernández et al., "New microstrip "wiggly-line" filters with spurious passband suppression," IEEE Transactions on Microwave Theory and Techniques, vol. 49, no. 9, pp. 1593-1598, 2001.

[4] J. García-García, F. Martín, F. Falcone et al., "Spurious passband suppression in microstrip coupled line band pass filters by means of split ring resonators," IEEE Microwave and Wireless Components Letters, vol. 14, no. 9, pp. 416-418, 2004.

[5] C.-F. Chen, T.-Y. Huang, and R.-B. Wu, "Design of microstrip bandpass filters with multiorder spurious-mode suppression,"
IEEE Transactions on Microwave Theory and Techniques, vol. 53, no. 12, pp. 3788-3793, 2005.

[6] Y.-M. Chen, S.-F. Chang, C.-C. Chang, and T.-J. Hung, "Design of stepped-impedance combline bandpass filters with symmetric insertion-loss response and wide stopband range," IEEE Transactions on Microwave Theory and Techniques, vol. 55, no. 10, pp. 2191-2199, 2007.

[7] C.-F. Chen, T.-Y. Huang, and R.-B. Wu, "Compact microstrip cross-coupled bandpass filters using miniaturized stepped impedance resonators," in Proceedings of the Asia-Pacific Microwave Conference (APMC '05), pp. 493-496, December 2005.

[8] T.-N. Kuo, W.-C. Li, C.-H. Wang, and C. H. Chen, "Widestopband microstrip bandpass filters using quarter-wavelength stepped-impedance resonators and bandstop embedded resonators," IEEE Microwave and Wireless Components Letters, vol. 18, no. 6, pp. 389-391, 2008.

[9] C.-H. Wang, P.-H. Deng, and C. H. Chen, "Coplanarwaveguide-fed microstrip bandpass filters with capacitively broadside-coupled structures for multiple spurious suppression," IEEE Transactions on Microwave Theory and Techniques, vol. 55, no. 4, pp. 768-775, 2007. 

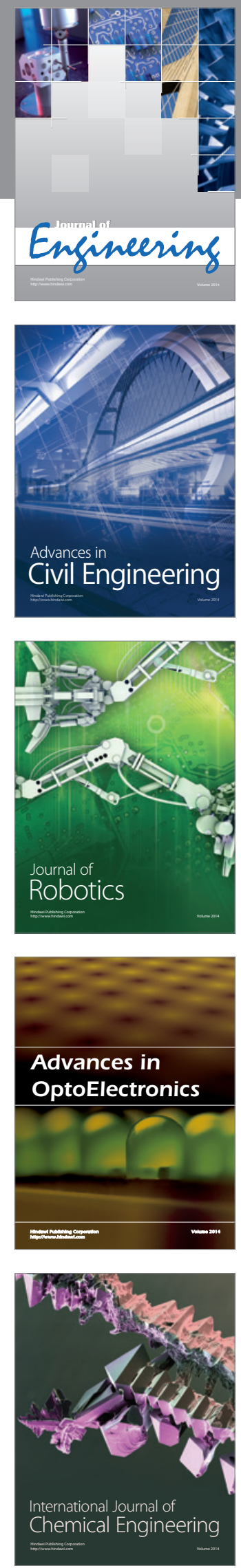

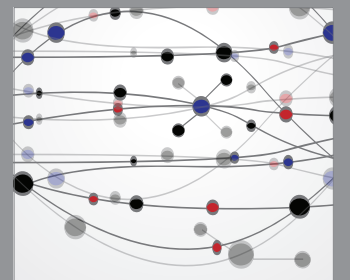

The Scientific World Journal
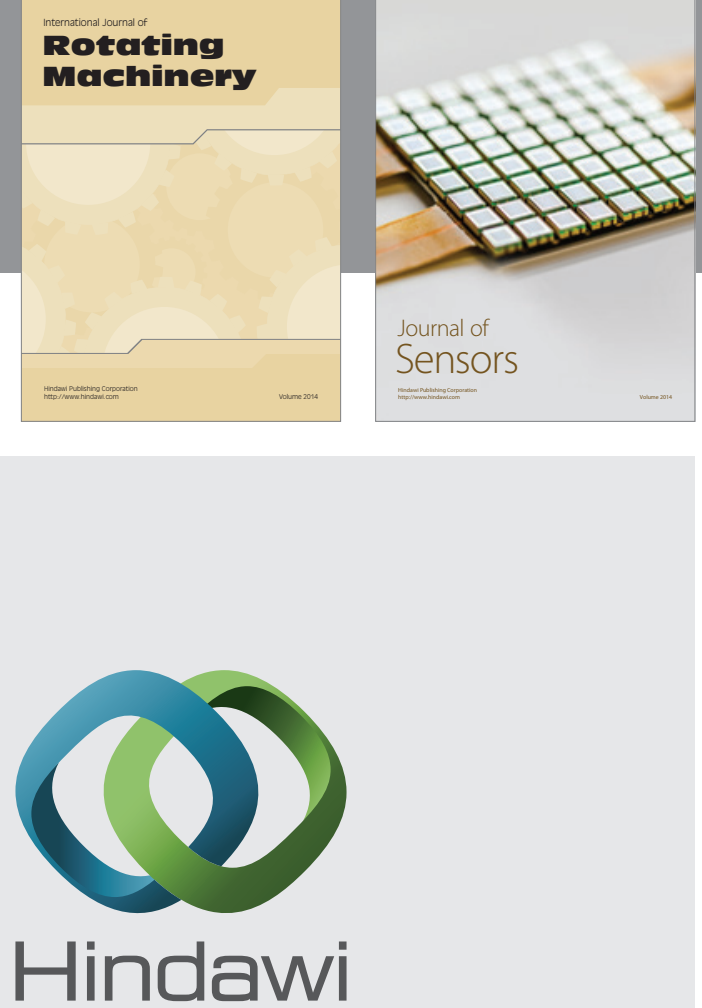

Submit your manuscripts at http://www.hindawi.com
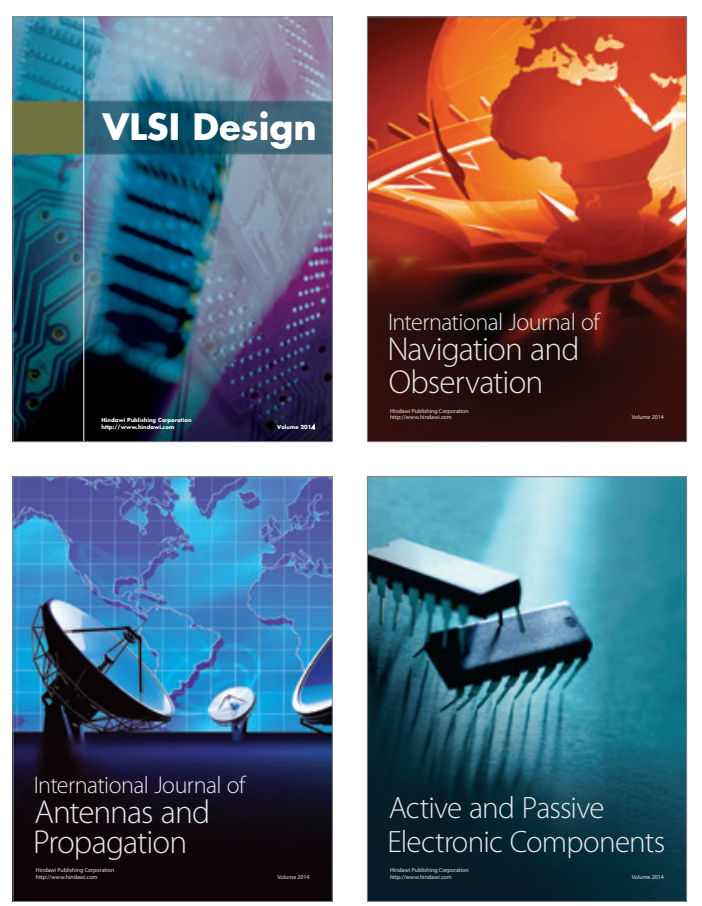
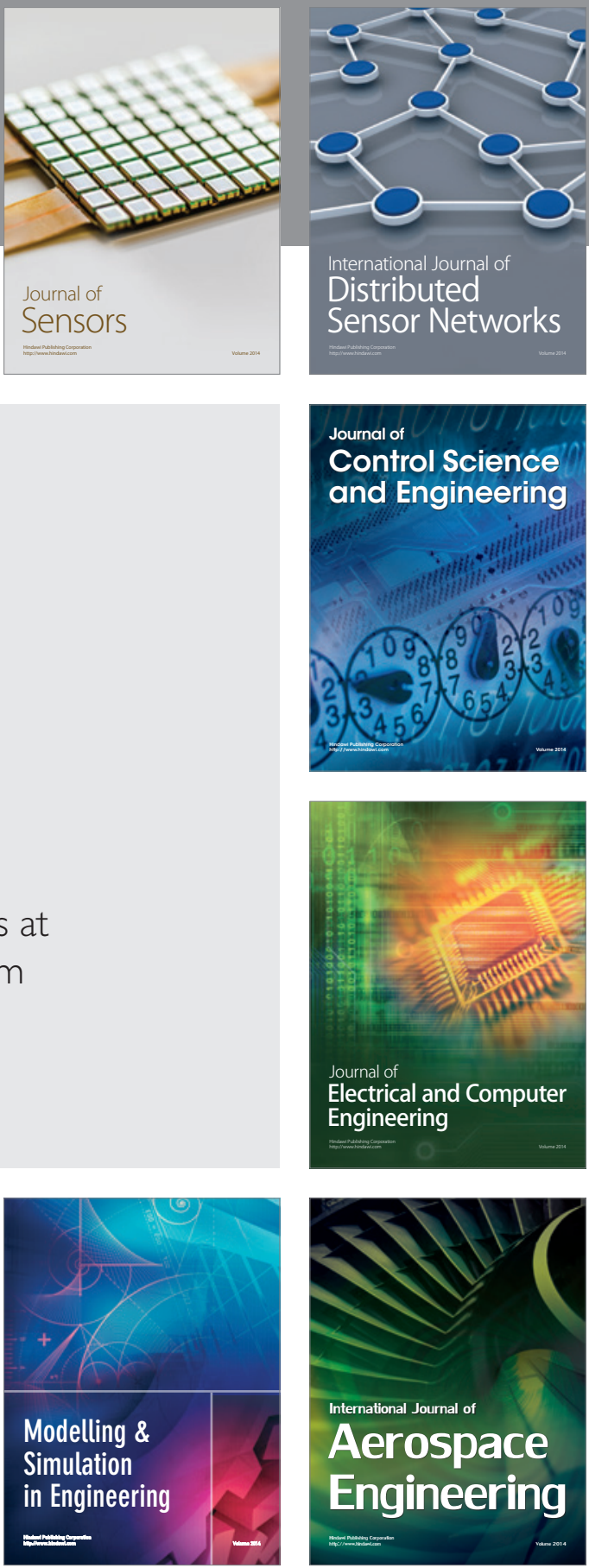

Journal of

Control Science

and Engineering
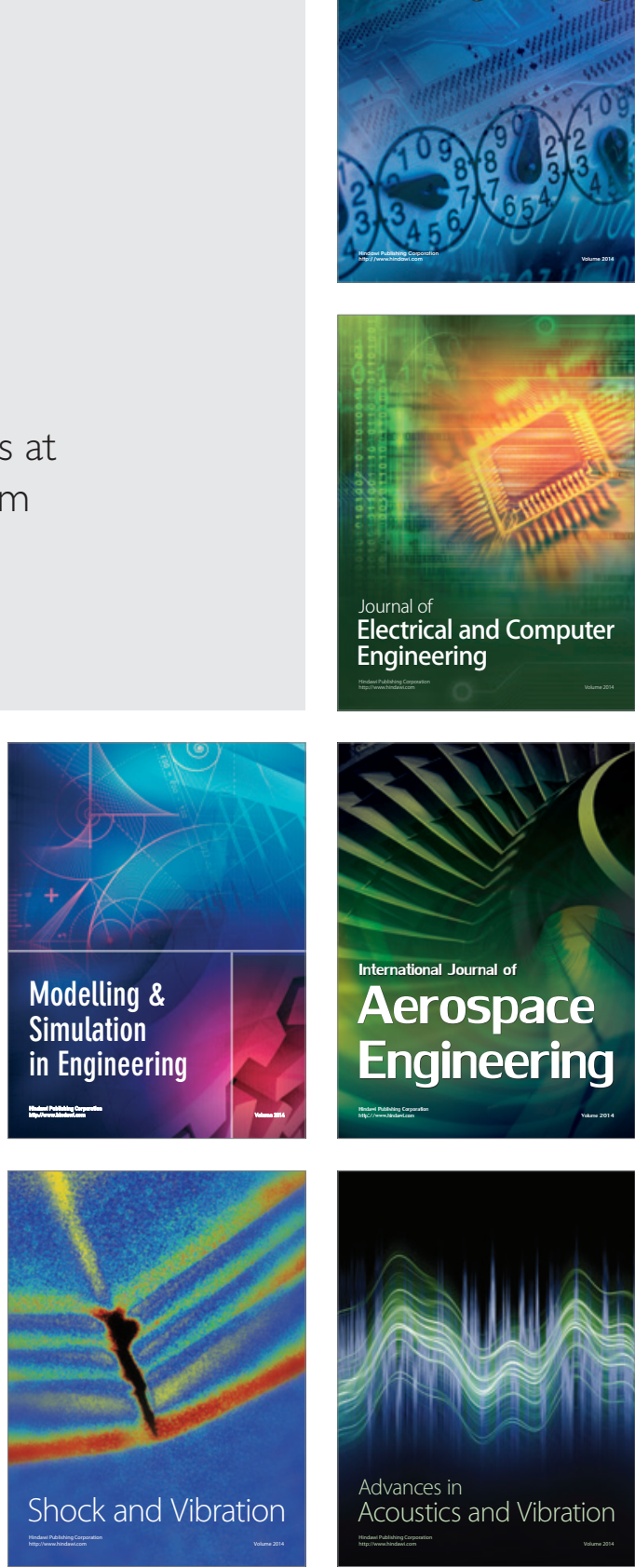